\title{
A Counterexample to the Quantizability of Modules
}

\section{Journal Article}

\section{Author(s):}

Willwacher, Thomas

Publication date:

2007

Permanent link:

https://doi.org/10.3929/ethz-b-000005102

\section{Rights / license:}

In Copyright - Non-Commercial Use Permitted

\section{Originally published in:}

Letters in Mathematical Physics 81(3), https://doi.org/10.1007/s11005-007-0179-6 


\title{
A Counterexample to the Quantizability of Modules
}

\author{
THOMAS WILLWACHER \\ Department of Mathematics, ETH Zurich, Rämistrasse 101, 8092 Zurich, Switzerland. \\ e-mail: thomas.willwacher@math.ethz.ch
}

Received: 17 July 2007 / Accepted: 1 August 2007

Published online: 21 August 2007

\begin{abstract}
Let a Poisson structure on a manifold $M$ be given. If it vanishes at a point $m$, the evaluation at $m$ defines a one dimensional representation of the Poisson algebra of functions on $M$. We show that this representation can, in general, not be quantized. Precisely, we give a counterexample for $M=\mathbb{R}^{n}$, such that: (i) The evaluation map at zero can not be quantized to a representation of the algebra of functions with product the Kontsevich product associated to the Poisson structure. (ii) For any formal Poisson structure extending the given one and still vanishing at zero up to second order in epsilon, (i) still holds. We do not know whether the second claim remains true if one allows the higher order terms in epsilon to attain nonzero values at zero.
\end{abstract}

Mathematics Subject Classifications (2000). 53D55, 53D17, 17 B63.

Keywords. coisoitropic submanifolds, Poisson sigma model, quantum modules.

\section{How to read this paper in ten minutes}

The busy reader can take the following shortcut:

1. Read Theorem 7 on page 270 for the main result.

2. Read Definition 2 if its statement is not clear.

3. Look at Equations (12) and the preceding enumeration for the definition of the counterexample.

\section{Introduction}

Let $M$ be a smooth $n$-dimensional manifold equipped with a Poisson structure $\pi$, making $C^{\infty}(M)$ a Poisson algebra with bracket $\{\cdot, \cdot\}$. In this paper, we will exclusively deal with the case $M=\mathbb{R}^{n}$. Kontsevich [3] has shown that one can always quantize this algebra, i.e., find an associative product $\star_{\pi}$ on $C^{\infty}(M)[[\epsilon]]$ such that for all $f, g \in C^{\infty}(M)$

$$
f \star_{\pi} g=f g+\frac{\epsilon}{2}\{f, g\}+O\left(\epsilon^{2}\right) \text {. }
$$

The author was partially supported by the Swiss National Science Foundation (grant 200020-105450). 
Furthermore, he showed that the set of such star products is, up to equivalence, in one to one correspondence with the set of formal Poisson structures on $M$ extending $\pi$.

DEFINITION 1. A formal Poisson structure $\pi_{\epsilon}$ is a formal bivector field $\pi_{\epsilon} \epsilon$ $\Gamma\left(\Lambda^{2} T M\right)[[\epsilon]]$ satisfying the Jacobi identity $\left[\pi_{\epsilon}, \pi_{\epsilon}\right]=0$, where $[\cdot, \cdot]$ is the Schouten-Nijenhuis bracket. We say that $\pi_{\epsilon}$ extends the Poisson structure $\pi \epsilon$ $\Gamma\left(\Lambda^{2} T M\right)$ if its $\epsilon^{0}$-component is $\pi$.

Let now $m \in M$ be a point and consider the evaluation map

$$
e v_{m}: C^{\infty}(M) \ni f \mapsto f(m) \in \mathbb{C} .
$$

It makes $\mathbb{C}$ into a $C^{\infty}(M)$-module, i.e., for all $f, g \in C^{\infty}(M)$, we have $e v_{m}(f g)=$ $e v_{m}(f) e v_{m}(g)$. The question treated in this paper is the following:

Main Question: Can one quantize the evaluation map $e v_{m}$ ?

By this we mean the following:

DEFINITION 2. Let $\star_{\pi_{\epsilon}}$ be the Kontsevich star product associated to the formal Poisson structure $\pi_{\epsilon}$ on $M$ and let $m \in M$ be an arbitrary point. A linear map

$$
\rho: C^{\infty}\left(\mathbb{R}^{n}\right) \rightarrow \mathbb{C}[[\epsilon]]
$$

will be called quantization of $e v_{m}$ if the following holds.

1. It has the form

$$
\rho(f)=f(m)+\epsilon \rho_{1}(f)+\epsilon^{2} \rho_{2}(f)+\cdots
$$

where the $\rho_{k}$ are differential operators evaluated at $m$. Concretely, this means in local coordinates that $\rho_{k}(f)=\sum_{I} c_{I} \frac{\partial f}{\partial x^{I}}(m)$, where the sum is over multiindices and the $c_{I}$ are constants, vanishing except for finitely many $I$.

2. For all $f, g \in C^{\infty}(M)$

$$
\rho\left(f \star_{\pi_{\epsilon}} g\right)=\rho(f) \rho(g)
$$

LEMMA 3. Let $\pi_{\epsilon}=\pi+O(\epsilon)$ and $m \in M$. If a quantization of ev exists, then $\pi(m)=0$, i.e., $\pi$ vanishes at $m$.

Proof. The $\epsilon^{1}$-component of the equation $\rho\left([f, g]_{\star}\right)=[\rho(f), \rho(g)]=0$ reads $\{f, g\}(m)=0$. Hence $\pi(m)=0$.

Remark. A similar calculation for a higher dimensional submanifold also yields the higher dimensional coisotropy condition. 
From now on we will assume that $\pi(m)=0$, or, equivalently, that $\{m\} \subset M$ is coisotropic. For details on coisotropic submanifolds see [2].

The above Main Question has been answered positively by Cattaneo and Felder in $[1,2]$, provided $\pi$ satisfies certain conditions. Adapted to our context, they proved the following theorem.

THEOREM 4. For any formal Poisson structure $\pi_{\epsilon}$ on $M=\mathbb{R}^{n}$ such that $\pi_{\epsilon}(m)=$ $0+O(\epsilon)$ for some $m \in M$, there exists a linear map $\tilde{\rho}: C^{\infty}(M) \rightarrow \mathbb{C}[[\epsilon]]$,

$$
\tilde{\rho}(f)=f(m)+\epsilon^{2} \tilde{\rho}_{2}(f)+\cdots 1
$$

where the $\tilde{\rho}_{k}$ are differential operators evaluated at $m$, that satisfies

$$
\tilde{\rho}\left(f \star_{\pi_{\epsilon}} g\right)=\tilde{\rho}(f) \tilde{\rho}(g)+A(f, g)
$$

for all $f, g \in C^{\infty}(M)$. Here $A=O\left(\epsilon^{2}\right)$ (the "anomaly") is a bidifferential operator evaluated at $m$.

Hence, if the "anomalous" term on the right-hand side of (2) vanishes, one sees that $\tilde{\rho}$ becomes a quantization of $e v_{m}$. The precise form of $A(f, g)$ is recalled in Section 1.2.

When $\pi_{\epsilon}(m)=0$ to all orders in $\epsilon$, the anomaly is actually at least of order $\epsilon^{3}$. Furthermore, we will later provide an example for which the $\epsilon^{3}$-term does not vanish.

A theorem similar to Theorem 4 above holds in the case of higher dimensional coisotropic submanifolds. There, anomaly terms will also occur in general. It is still an open question whether the vanishing of these terms is merely a removable technical condition or a fundamental obstruction to quantizability. Our paper gives a partial answer to this question in the simplest possible case.

\subsection{QUANTIZATION OF MODULES}

In this section we briefly recall the construction of Cattaneo and Felder [2] leading to Theorem 4. We throughout assume familiarity with the construction of Kontsevich's star product [3]. The map $\tilde{\rho}$ of Theorem 4 has the explicit form

$$
\tilde{\rho}(f)=\sum_{\Gamma} \tilde{w}_{\Gamma} D_{\Gamma}(f) .
$$

The sum is over all Kontsevich graphs with one type II vertex (associated to $f$ ). ${ }^{2}$ The differential operator $D_{\Gamma}$ is constructed exactly as it is constructed for

\footnotetext{
${ }^{1}$ Note the absence of the $\epsilon^{1}$ term.

${ }^{2}$ Recall that in a Kontsevich graph, there are two kinds of vertices. Type I or "aerial" vertices represent one copy of the Poisson structure $\pi$, whereas type II vertices are associated to the functions one intends to multiply.
} 
Kontsevich's star product. The weights $\tilde{w}_{\Gamma}$ are given by the integral formula

$$
\tilde{w}_{\Gamma}=\int_{\tilde{C}_{\Gamma}} \tilde{\omega}_{\Gamma} .
$$

Here the space $\tilde{C}_{\Gamma}$ is the Cattaneo-Felder configuration space. It is (a compactification of) the space of all embeddings of the vertex set of $\Gamma$ into the first quadrant, modulo rescalings. Furthermore, the embeddings are required to map the type II vertex into the real axis. Similar to the Kontsevich case, the weight form $\tilde{\omega}_{\Gamma}$ is defined as a product of one-forms, one for each edge in the edge set $E(\Gamma)$ of $\Gamma$.

$$
\tilde{\omega}_{\Gamma}= \pm \bigwedge_{e \in E(\Gamma)} d \phi\left(z_{e_{1}}, z_{e_{2}}\right) .
$$

Here the edge $e$ in the product is understood to point from the vertex $e_{1}$ that is mapped to $z_{e_{1}}$ to the vertex $e_{2}$, that is mapped to $z_{e_{2}}$.

The precise expression for the angle form $d \phi\left(z_{1}, z_{2}\right)$ will never be needed, but we will use the following facts about its boundary behaviour in the Appendix:

1. If $z_{1}$ lies on the real axis or $z_{2}$ on the imaginary axis, $d \phi\left(z_{1}, z_{2}\right)$ vanishes.

2. If $z_{1}$ and $z_{2}$ both come close to each other and to a point on the positive real axis, $d \phi\left(z_{1}, z_{2}\right)$ approaches Kontsevich's angle form.

3. If $z_{1}$ and $z_{2}$ both come close to each other and to a point on the positive imaginary axis, $d \phi\left(z_{2}, z_{1}\right)$ approaches Kontsevich's angle form, i.e., $d \phi\left(z_{1}, z_{2}\right)$ approaches Kontsevich's form after reversal of the edge direction.

\subsection{CONSTRUCTION OF THE ANOMALY}

The anomaly $A(f, g)$ in Theorem 4 can be computed by the formula

$$
A(f, g)=\sum_{\Gamma} \tilde{w}_{\Gamma} D_{\Gamma}(f, g)
$$

Here the sum is over all anomaly graphs. Such a graph is a Kontsevich graph, but with a third kind of vertices, which we call anomalous or type III vertices. An anomaly graph is required to contain at least one such type III vertex. These anomalous vertices have exactly 2 outgoing edges.

The weight $\tilde{w}_{\Gamma}$ is computed just as the Cattaneo-Felder weight, but with the type III vertices constraint to be mapped to the imaginary axis.

The computation of $D_{\Gamma}$ also remains the same as before, but one has to specify which bivector fields to associate with the new type III vertices. In local coordinates $x^{i}, i=1, \ldots, n$, the components of this bivector field will be denoted $\epsilon \pi_{a}^{i j}$. 3 It is in turn given as a sum of graphs.

$$
\pi_{a}^{i j}=\sum_{\Gamma} a_{\Gamma} D_{\Gamma}\left(x^{i}, x^{j}\right)
$$

\footnotetext{
${ }^{3}$ The " $a$ " in $\pi_{a}^{i j}$ is not an index, just a label.
} 
Here the sum is over all Cattaneo-Felder graphs with 2 type II vertices and $D_{\Gamma}$ is again defined as in the Cattaneo-Felder case before. However, the weights $a_{\Gamma}$ are computed by the following algorithm:

1. Delete the type II vertices in $\Gamma$ and all their adjacent edges.

2. Reverse the direction of all edges.

3. Compute the Kontsevich weight of the resulting graph.

With this anomalous vertex, one can construct two kinds of graphs that yield $O\left(\epsilon^{3}\right)$-contributions:

1. The graph with only one vertex, which is anomalous. It yields the contribution proportional to $\epsilon \pi_{a}^{i j}$ to the anomaly.

2. The graphs with one type I and one anomalous vertex as shown in Figure 1. Together, they yield a symmetric contribution to the anomaly.

We will use the following notation for the parts of $A(f, g)$ of various orders in $\epsilon:^{4}$

$$
\left.A(f, g)=\epsilon^{2} A_{2}^{i j}\left(\partial_{i} f\right)\left(\partial_{j} g\right)+\epsilon^{3} A_{3}^{i j}\left(\partial_{i} f\right)\left(\partial_{j} g\right)+\epsilon^{3} \text { (symm. in } f, g\right)+O\left(\epsilon^{4}\right)
$$

Here $A_{2}^{i j}=\pi_{1}^{i j}$ and $A_{3}^{i j}$ are antisymmetric. The contribution to $A_{3}^{i j}$ comes from the two graphs in Figure 2, with $\pi^{\prime} s$ attached to the vertices of the left graph and a $\pi_{2}$ attached to the vertex of the right graph. Note also that if $\pi_{\epsilon}(0)=0$ to all orders in $\epsilon$, then the contribution of the right graph vanishes and $A_{2}^{i j}=0$.

Remark 5. (Linear Poisson structures) It is easily seen that, if the Poisson structure $\pi_{\epsilon}=\pi$ is linear, i.e., if $M$ is the dual of a Lie algebra, the anomalous vertex $\pi_{a}$ vanishes [2]. This is because any contributing graph with $n$ vertices will contain $2 n-2$ edges. But for a graph $\Gamma$ with different numbers of vertices and edges, $D_{\Gamma}=0$ by power counting. Hence a contribution will not arise unless $n=2$. But the weight of the only possible graph with 2 vertices is 0 by one of Kontsevich's lemmas [3], i.e., a reflection argument.

This also implies that the anomaly can be removed whenever the Poisson structure is linearizable. At least formally, the Poisson structure can be linearized whenever the second Lie algebra cohomology of the Lie algebra defined by the linear order, with values in the symmetric algebra, vanishes. For example, this is true for semisimple Lie algebras. See [5] for details.

Remark 6. (Higher order Poisson structures) As pointed out to the author by A. S. Cattaneo and G. Felder, the anomaly also vanishes whenever the linear order of $\pi$ does. This is shown by power counting: Each vertex in an anomaly graph comes with two edges (derivatives) and is of at least quadratic order. But two edges have to be external and do not contribute derivatives, so the result vanishes when evaluated at $m$.

\footnotetext{
${ }^{4}$ Here and in the following summation over repeated indices is implicit.
} 

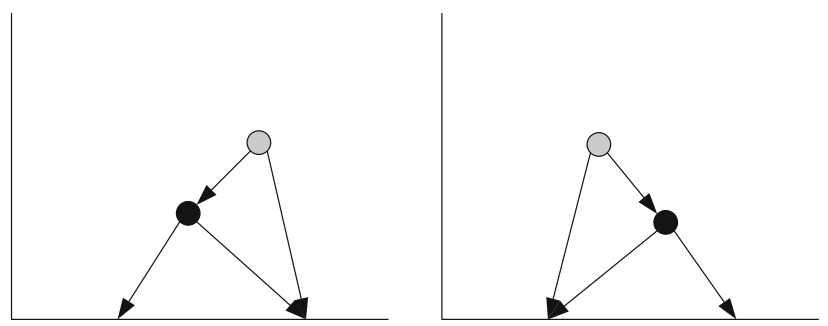

Figure 1. The two anomaly graphs contributing to the symmetric $\epsilon^{3}$ part of the anomaly $\mathrm{A}(\mathrm{f}, \mathrm{g})$. The black vertex is a "normal" type I vertex, corresponding to $\pi$. The grey vertex is an anomalous vertex corresponding to $\pi_{a}$. To this order, $\pi_{a}=\pi_{1}$.
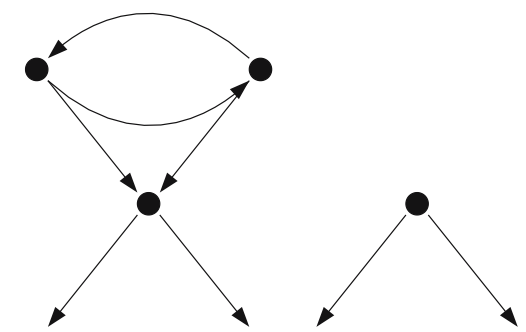

Figure 2. The graphs accounting for the $\epsilon^{2}$ and $\epsilon^{3}$ contributions to the anomaly vertex $\pi_{a}$. The right graph only contributes if $\pi_{\epsilon}(0) \neq 0$.

\section{Statement of the Main Theorem}

THEOREM 7. There exists a Poisson structure $\pi$ on $\mathbb{R}^{n}$ vanishing at 0 , s.t. no quantization of $e v_{0}$ exists for the Kontsevich star product associated to $\pi$. Furthermore, this also holds for any formal Poisson structure $\pi_{\epsilon}$ extending $\pi$

$$
\pi_{\epsilon}=\pi+\epsilon \pi_{1}+\cdots
$$

as long as $\pi_{\epsilon}(0)=0$.

Remark 8. Placing this theorem into a more general context, this means that the $C^{\infty}(M)$-module structure on a coisotropic submanifold can not always be quantized to a module structure for $\left(C^{\infty}(M)[[\epsilon]], \star\right)$, where $\star$ is the usual Kontsevich product. Hence this theorem answers the Main Question negatively. However, the new question arises whether one can relax the condition $\pi_{\epsilon}(0)=0$ to $\pi_{\epsilon}(0)=0+$ $O(\epsilon)$. A complete answer to this new question we cannot give, only some more hints, see [6].

\section{The Proof}

Without loss of generality we can use the following ansatz for $\rho$.

$$
\rho=\tilde{\rho}+\phi
$$


Here $\tilde{\rho}$ is the map of Theorem 4 and $\phi$ has the form $\phi=\epsilon \phi_{1}+\epsilon^{2} \phi_{2}+\cdots$. Our goal, and content of the next sections, is to find the lowest order restrictions on $\phi$ coming from the requirement of $\rho$ being a quantum module map, i.e., Equation (1). Concretely, the requirement is

$$
\phi(f \star g)+A(f, g)=\tilde{\rho}(f) \phi(g)+\phi(f) \tilde{\rho}(g)+\phi(f) \phi(g) .
$$

Here we simply inserted (3) into (1) and used (2). To order $\epsilon^{0}$ this equation is obviously satisfied.

\subsection{ORDER $\epsilon^{1}$}

The $\epsilon^{1}$ part of Equation (4) reads

$$
\phi_{1}(f g)=\phi_{1}(f) g(0)+f(0) \phi_{1}(g) .
$$

Choosing $f, g$ both constant we see that the zeroth derivative part of $\phi_{1}$ has to vanish. Choosing $f$ and $g$ both linear the right-hand side vanishes and hence the second-derivative contribution to $\phi_{1}$ has to vanish. Picking $f$ quadratic and $g$ linear we see that the third-derivative part of $\rho_{1}$ must vanish and similarly that all higher derivative parts must vanish as well. Hence

$$
\phi_{1}(f)=D_{1}^{k}\left(\partial_{k} f\right)(0)
$$

for some constants $D_{1}^{k}, k=1, \ldots, n$.

\subsection{ORDER $\epsilon^{2}$}

We will separately consider the contributions symmetric and antisymmetric in $f$, $g$. The antisymmetric contribution reads

$$
\phi_{1}(\{f, g\})=0 .
$$

Note that if $\pi_{\epsilon}(0)=0$, then $A_{2}(f, g)=0$. The left-hand side of (7) is zero if $f$ or $g$ contains no linear part. Hence it suffices to treat the case where $f$ and $g$ are both linear. Then the equation becomes

$$
D_{1}^{k}\left(\partial_{k} \pi^{i j}\right)(0)=0
$$

where $\pi^{i j}=\left\{x^{i}, x^{j}\right\}$ are the components of $\pi$ w.r.t. the standard coordinates $\left\{x^{i}\right\}_{i=1, . ., n}$.

The symmetric part yields the constraint

$$
\phi_{2}(f g)=\phi_{1}(f) \phi_{1}(g)+\phi_{2}(f) g(0)+f(0) \phi_{2}(g) .
$$

Picking $f, g$ linear we see that the second derivative part of $\phi_{2}$ must be

$$
\phi_{2}^{(2)}=\frac{1}{2} D_{1}^{i} D_{1}^{j} \partial_{i} \partial_{j}
$$


Inserting this back into (9) we obtain the same constraint equation for the remaining parts of $\phi_{2}$ as we had found for $\phi_{1}$ in Equation (5). By the same logic as there we can hence deduce that

$$
\phi_{2}=\frac{1}{2} D_{1}^{i} D_{1}^{j} \partial_{i} \partial_{j}+D_{2}^{i} \partial_{i}
$$

for some yet undetermined constants $D_{2}^{i}, i=1, \ldots, n$. Here, all derivatives are implicitly understood to be evaluated at zero, e.g.,

$$
\partial_{i} \partial_{j}(f):=\left(\partial_{i} \partial_{j} f\right)(0) .
$$

Remark 9. Note that the calculations presented so far are valid for any formal Poisson structure

$$
\pi_{\epsilon}=\pi+\epsilon \pi_{1}+\cdots
$$

as long as it vanishes at 0 , i.e., the higher order terms do not contribute to the first two orders in $\epsilon$ of Equation (4).

\subsection{ORDER $\epsilon^{3}$}

We will only need to consider the antisymmetric part and linear $f=x^{i}$ and $g=x^{j}$ in Equation (4). In this case the $\epsilon^{3}$ part of the equation becomes

$$
D_{1}^{k} \partial_{k} \pi_{1}^{i j}+\frac{1}{2} D_{1}^{k} D_{1}^{l} \partial_{k} \partial_{l} \pi^{i j}+D_{2}^{k} \partial_{k} \pi^{i j}+2 A_{3}^{i j}=0 .
$$

The first term is the contribution of the $\epsilon^{1}$-term in the formal Poisson structure $\pi_{\epsilon}$. It is absent if we consider $\pi_{\epsilon}=\pi$. To derive the above formula we used the following.

- The right-hand side of (4) is obviously symmetric in $f, g$, hence all contributions come from the left-hand side.

- The Kontsevich product satisfies

$$
\left[x^{i}, x^{j}\right]_{\star}=\epsilon \pi^{i j}+\epsilon^{2} \pi_{1}^{i j}+O\left(\epsilon^{3}\right) .
$$

Hence, using (8) we obtain

$$
\begin{aligned}
\phi\left(\left[x^{i}, x^{j}\right]_{\star}\right) & =\epsilon^{3} \phi_{1}\left(\pi_{1}^{i j}\right)+\epsilon^{3} \phi_{2}\left(\pi^{i j}\right)+O\left(\epsilon^{4}\right) \\
& =\epsilon^{3}\left(D_{1}^{k} \partial_{k} \pi_{1}^{i j}+\frac{1}{2} D_{1}^{k} D_{1}^{l} \partial_{k} \partial_{l} \pi^{i j}+D_{2}^{k} \partial_{k} \pi^{i j}\right)+O\left(\epsilon^{4}\right)
\end{aligned}
$$




\subsection{THE COUNTEREXAMPLE}

In this section we present a $\pi$ such that there are no constants $D_{1,2}^{k}$ satisfying (8) and (11) for $\pi_{1}=0$. This will prove the first part of Theorem 7 .

For this, the following data are needed:

1. Some finite dimensional semisimple Lie algebra $\mathfrak{g}$ with structure coefficients $g_{k}^{i j}$ in some basis $\left\{x^{i}\right\}$. We denote by $K^{i j}$ its Killing form and by $K_{i j}$ its inverse.

2. Some finite dimensional Lie algebra $\mathfrak{h}$ such that its second cohomology group $H^{2}(\mathfrak{h}, \mathbb{C}) \neq\{0\}$. Denote its structure coefficients $h_{c}^{a b}$.

3. A non-trivial $C \in H^{2}(\mathfrak{h}, \mathbb{C})$, with coefficients $C^{a b}$ in some basis $\left\{y^{a}\right\}$.

EXAMPLE 10. The simplest possible choice would be $\mathfrak{g}=\mathfrak{s o}(3), \mathfrak{h}=\mathbb{R}^{2}$ as Abelian Lie algebra and $C^{12}=-C^{21}=1, C^{11}=C^{22}=0$.

The Poisson structure will reside in

$$
\mathbb{R}^{n} \cong(\mathfrak{g} \oplus \mathfrak{h})^{*} \cong \mathfrak{g}^{*} \oplus \mathfrak{h}^{*}
$$

where $n=\operatorname{dim} \mathfrak{g}+\operatorname{dim} \mathfrak{h}$. We use as coordinate functions the above basis $x^{i}$ and $y^{a}$. Then one can define

$$
\pi^{i j}=x^{k} g_{k}^{i j} \quad \pi^{a i}=0 \quad \pi^{a b}=y^{c} h_{c}^{a b}+\Psi(x) C^{a b}
$$

where $\Psi(x)=K_{i j} x^{i} x^{j}$ is the quadratic Casimir in $S^{2} \mathfrak{g}$.

LEMMA 11. The above $\pi$ defines a Poisson structure on $\mathbb{R}^{n}$.

Proof. Denote by $\pi^{(1)}, \pi^{(2)}$ the linear and quadratic parts of $\pi$, respectively. We need to show that

$$
[\pi, \pi]=\left[\pi^{(1)}, \pi^{(1)}\right]+2\left[\pi^{(1)}, \pi^{(2)}\right]+\left[\pi^{(2)}, \pi^{(2)}\right]=0
$$

where $[\cdot, \cdot]$ denotes the Schouten-Nijenhuis bracket. The linear part of the equation, i.e., $\left[\pi^{(1)}, \pi^{(1)}\right]=0$ is satisfied since $\mathfrak{g} \oplus \mathfrak{h}$ is a Lie algebra. The cubic part $\left[\pi^{(2)}, \pi^{(2)}\right]=0$ is trivially satisfied since all vector fields $\frac{\partial}{\partial y^{a}}$ commute with all $x^{i}$.

The quadratic part $\left[\pi^{(1)}, \pi^{(2)}\right]=0$ is equivalent to

$$
\left\{f,\{g, h\}_{2}\right\}_{1}+\left\{f,\{g, h\}_{1}\right\}_{2}+\text { cycl. }=0
$$

for all linear $f, g, h \in C^{\infty}\left(\mathbb{R}^{n}\right)$. Here $\{\cdot, \cdot\}_{1,2}$ are the Poisson brackets of the Poisson structures $\pi^{(1)}$ and $\pi^{(2)}$, respectively. By trilinearity, we can separately consider the following cases. 
- If at least two of the $f, g, h$ are functions of the $x^{i}$,s only, the expression trivially vanishes since the set of these functions is closed under $\{\cdot, \cdot\}_{1}$, and furthermore $\left\{x^{i}, \cdot\right\}_{2}=0$.

- If $f=y^{a}, g=y^{b}, h=y^{c}$ the only contributing term is the second, i.e., $\Psi(x) C^{a d} h_{d}^{b c}+$ cycl., which vanishes by the cocycle property of $C^{a b}$.

- The remaining case is $f=x^{i}, g=y^{a}, h=y^{b}$, leading to $\left\{x^{i}, \Psi(x)\right\}_{1} C^{a b}$ since $\left\{x^{i}, y^{a, b}\right\}_{1,2}=0$. This term vanishes because the Casimir element is Poisson central.

Knowing that $\pi$ defines a Poisson structure, we can continue the proof of the main theorem. This will be done in two lemmas.

LEMMA 12. The anomaly $A_{3}(f, g)$ associated to $\pi$ as in Theorem 4 is a nonzero multiple of

$$
C(f, g):=C^{a b}\left(\partial_{a} f\right)(0)\left(\partial_{b} g\right)(0) .
$$

Proof. The anomaly is given by the left graph, say $\Gamma$, of Figure 2 . It will be shown in the Appendix that its weight $\tilde{w}_{\Gamma}$ is nonzero. The associated bidifferential operator (applied to functions $f, g$ ) is given by

$$
\begin{aligned}
D_{\Gamma}(f, g) & =K^{\alpha \beta}\left(\partial_{\alpha} \partial_{\beta} \pi^{\gamma \delta}\right)\left(\partial_{\gamma} f\right)\left(\partial_{\delta} g\right)=K^{i j}\left(\partial_{i} \partial_{j} \Psi(x)\right) C^{a b}\left(\partial_{a} f\right)\left(\partial_{b} g\right) \\
& =2 K^{i j} K_{i j} C^{a b}\left(\partial_{a} f\right)\left(\partial_{b} g\right)=2(\operatorname{dim} \mathfrak{g}) C^{a b}\left(\partial_{a} f\right)\left(\partial_{b} g\right)
\end{aligned}
$$

Here and in the following we adopt the convention that Greek indices refer to a basis

$$
\xi^{\alpha}= \begin{cases}x^{\alpha} & \text { for } \alpha=1, \ldots, \operatorname{dim} \mathfrak{g} \\ y^{\alpha-\operatorname{dim} \mathfrak{g}} & \text { for } \alpha=\operatorname{dim} \mathfrak{g}+1, \ldots, n\end{cases}
$$

of $\mathfrak{g} \oplus \mathfrak{h}$, and are summed over $1, \ldots, n$ if repeated. In contrast the roman indices $i, j$ label the basis $x^{i}$ of $\mathfrak{g}$ only and are summed over $1, \ldots, \operatorname{dim} \mathfrak{g}$ if repeated. Similarly, the roman indices $a, b$ refer to the basis $y^{a}$ of $\mathfrak{h}$ only and are summed over $\operatorname{dim} \mathfrak{g}+1, \ldots, n$.

LEMMA 13. For the above $\pi$ and $\pi_{1}=0$, Equations (8) and (11) cannot be solved simultaneously.

Proof. Since $\mathfrak{g}$ is semisimple $[\mathfrak{g}, \mathfrak{g}]=\mathfrak{g}$ and Equation (8) implies that $D_{1}^{i}=0$ for all $i=1, \ldots, \operatorname{dim}(\mathfrak{g})$. But then also $D_{1}^{\alpha} D_{1}^{\beta}\left(\partial_{\alpha} \partial_{\beta} \pi^{\gamma \delta}\right)=0$ since $\pi$ contains no part quadratic in the $y^{a}$. Hence Equation (11) becomes

$$
D_{2}^{\alpha}\left(\partial_{\alpha} \pi^{i j}\right)(0)=-2 A_{3}^{i j}=0 \quad D_{2}^{\alpha}\left(\partial_{\alpha} \pi^{a b}\right)(0)=-2 A_{3}^{a b} \propto C^{a b}
$$

Inserting the expression for $\pi$ we obtain from the second equation $D_{2}^{c} h_{c}^{a b} \propto C^{a b}$ stating that $C^{a b}$ is a coboundary. Hence, by choice of $C^{a b}$, this equation cannot be solved. Thus the lemma and the first part of Theorem 7 is proven. 


\subsection{A SPECIALIZED COUNTEREXAMPLE}

We finally turn to the more general case where $\pi_{1} \neq 0$, but still $\pi_{\epsilon}(0)=0$. The construction in this case runs as above, but we make the special choice $\mathfrak{h}=\mathfrak{k} \oplus \mathfrak{k}$, where $\mathfrak{k}$ is the (unique) non-abelian two dimensional Lie algebra. Its cohomology groups are computed in the Appendix. There is, up to normalization, only one non-trivial cocycle we can pick, namely $\omega$ as defined in Equation (16) in the Appendix. We will call the resulting Poisson structure $\pi$. The proof of the main Theorem 7 will then be finished by proving the following lemma.

LEMMA 14. For any formal Poisson structure $\pi_{\epsilon}$ extending the $\pi$ constructed above, for which $\pi_{\epsilon}(0)=0$, Equation (8) and (11) cannot be solved simultaneously.

Proof. By the previous proof it will be sufficient to show that we cannot pick $\pi_{1}$ and $D_{1}^{a}$ such that

$$
D_{1}^{c} \partial_{c} \pi_{1}^{a b}(0)-\text { (const.) } C^{a b}
$$

becomes (the coefficients of) an exact element of $H^{2}(\mathfrak{h}, \mathbb{R})$. The $\epsilon^{1}$ part of the Jacobi equation for the Poisson structure $\pi_{\epsilon} \operatorname{reads}\left[\pi, \pi_{1}\right]=0$. Considering only the linear part we have

$$
\left[\pi^{(1)}, \pi_{1}^{(1)}\right]=0 \text {. }
$$

where $\pi^{(1)}, \pi_{1}^{(1)}$ are the linear parts of $\pi$ and $\pi_{1}$ respectively. Note that we used here that the constant part $\pi_{1}^{(0)}=0$. Equation (14) means that $\pi_{1}^{(1)}$ defines a 2-cocycle of $\mathfrak{g} \oplus \mathfrak{h}$ with values in the adjoint module.

Equivalently, by projecting on the invariant submodules $\mathfrak{g}$ or $\mathfrak{h}$, one has two 2-cocycles, with values in the $\mathfrak{g} \oplus \mathfrak{h}$-modules $\mathfrak{g} \otimes \mathbb{R}$ and $\mathbb{R} \otimes \mathfrak{h}$ respectively. Here $\mathbb{R}$ is always understood as equipped with the trivial module structure, and $\mathfrak{g}$, $\mathfrak{h}$ with the adjoint structures.

The first 2-cocycle is irrelevant to us since it does not occur in (13), for $D_{1}^{i}=0$. The second 2-cocycle defines a cohomology class, say $\left[\pi_{1}\right]$, in $H^{2}(\mathfrak{g} \oplus \mathfrak{h}, \mathfrak{h})$. Equation (8) means that also $D_{1}^{c}$ is a cocycle and defines a cohomology class $\left[D_{1}\right] \in H^{0}\left(\mathfrak{g} \oplus \mathfrak{h}, \mathfrak{h}^{*}\right)$. The triviality of (13) implies that the cup product of the two cohomology classes above would have to satisfy

$$
\left[D_{1}\right] \cup\left[\pi_{1}\right] \neq 0
$$

But from the formulas of Künneth and Whitehead it follows that

$$
H^{2}(\mathfrak{g} \oplus \mathfrak{h}, \mathfrak{h}) \cong H^{0}(\mathfrak{g}, \mathbb{R}) \otimes H^{2}(\mathfrak{h}, \mathfrak{h}) \cong H^{2}(\mathfrak{h}, \mathfrak{h})
$$

Furthermore, as shown in the Appendix, $H^{2}(\mathfrak{h}, \mathfrak{h})=\{0\}$. Hence $\left[\pi_{1}\right]=0$ and Equation (15) can not be satisfied. Thus the lemma and Theorem 7 is proven. 


\section{Acknowledgements}

The author wants to thank his advisor Prof. G. Felder for his continuous support and patient explanation of most of the material presented here. Furthermore, the computation of weights in Appendix B is mainly due to G. Felder and A. S. Cattaneo, the author merely filled in some details.

\section{Appendix}

\section{A. COHOMOLOGY OF $\mathfrak{k}$ AND $\mathfrak{k} \oplus \mathfrak{k}$}

The Lie algebra $\mathfrak{k}$ is defined as the vector space $\mathbb{R}^{2}$ with the bracket

$$
\left[e_{1}, e_{2}\right]=e_{2}
$$

where $e_{1,2}$ are the standard basis vectors.

\section{LEMMA 15.}

$$
H^{0}(\mathfrak{k}, \mathbb{R}) \cong H^{1}(\mathfrak{k}, \mathbb{R}) \cong \mathbb{R}
$$

All other cohomology groups with values in $\mathbb{R}$ vanish. A representative of the equivalence class spanning $H^{1}(\mathfrak{k}, \mathbb{R})$ is $l: e_{1} \mapsto 1, e_{2} \mapsto 0$.

Proof. It is clear by antisymmetry that $H^{n}(\mathfrak{k}, \mathbb{C})=\{0\}$ for $n>2$ and also that any 2 -cochain is a cocycle. There is only one 2-cochain (up to a factor) and it is a coboundary since $c: e_{j} \mapsto \delta_{j, 2}$ satisfies $c\left(\left[e_{1}, e_{2}\right]\right)=1$. Finally, any 1-cocycle must vanish on $[\mathfrak{k}, \mathfrak{k}]=\mathbb{R} e_{2}$. Hence it is clear that the map $l$ defined in the lemma spans the space of 1-cocycles.

LEMMA 16. All cohomology groups of $\mathfrak{k}$ with values in $\mathfrak{k}$ vanish, i.e.,

$$
H^{p}(\mathfrak{k}, \mathfrak{k})=\{0\} \forall p .
$$

Proof. There is no central element in $\mathfrak{k}$, hence $H^{0}(\mathfrak{k}, \mathfrak{k})=\{0\}$. The cocycle condition for some $l: \mathfrak{k} \rightarrow \mathfrak{k}$ reads

$$
\begin{aligned}
(d l)\left(e_{1}, e_{2}\right) & =l\left(\left[e_{1}, e_{2}\right]\right)-\left[l\left(e_{1}\right), e_{2}\right]-\left[e_{1}, l\left(e_{2}\right)\right]=l\left(e_{2}\right)-e_{2} l_{1}\left(e_{1}\right)-e_{2} l_{2}\left(e_{2}\right) \\
& =e_{1} l_{1}\left(e_{2}\right)-e_{2} l_{1}\left(e_{1}\right) \stackrel{!}{=} 0
\end{aligned}
$$

where $l(\cdot)=e_{1} l_{1}(\cdot)+e_{2} l_{2}(\cdot)$. Hence $l_{1}(\cdot) \equiv 0$. But then

$$
l(\cdot)=\left[\cdot, l_{2}\left(e_{1}\right) e_{2}-l_{2}\left(e_{2}\right) e_{1}\right]
$$

and hence $l$ is exact and $H^{1}(\mathfrak{k}, \mathfrak{k})=\{0\}$. That $H^{p}(\mathfrak{k}, \mathfrak{k})=\{0\}$ for $p \geq 2$ follows as in the proof of the previous lemma. 
We now consider the direct sum $\mathfrak{h}=\mathfrak{k} \oplus \mathfrak{k}$. We denote the standard basis by $e_{1}, \ldots, e_{4}$. So, e.g., $\left[e_{3}, e_{4}\right]=e_{4}$. Künneth's formula and the above lemmas tell us the following:

$-H^{2}(\mathfrak{h}, \mathbb{C})$ is spanned by

$$
\omega: e_{1} \wedge e_{3} \mapsto 1
$$

with all other components vanishing.

$-H^{2}(\mathfrak{h}, \mathfrak{h})=\{0\}$.

\section{B. NONVANISHING OF THE 2-WHEEL GRAPH CONTRIBUTING TO THE ANOMALY}

One still needs to show that the weight of the left graph in Figure 2 is nonzero. We will actually compute the weights of all wheel graphs. Instead of defining "wheel graph", we refer to Figure 3, from which the definition should be clear. To compute the weights, we need the following result interesting in its own right.

PROPOSITION 17. Let $\mathfrak{g}$ be a Lie algebra and equip its dual space with the canonical Poisson structure. Let $e v_{0}$ be the evaluation map at zero and $\rho$ its quantization according to Cattaneo and Felder. Let $D: S \mathfrak{g} \rightarrow S \mathfrak{g}$ be the map

$$
D=\operatorname{det}^{\frac{1}{2}}\left(\frac{\sinh \left(a d_{\partial} / 2\right)}{a d_{\partial} / 2}\right)=\exp \left(\sum_{n \geq 1} \frac{B_{2 n}}{4 n(2 n) !} \operatorname{tr}\left(a d_{\partial}^{2 n}\right)\right)
$$

with $B_{j}$ the $j$ th Bernoulli number. ${ }^{5}$ Then

$$
\rho=e v_{0} \circ D^{-1} \text {. }
$$

Proof. The map $D$ intertwines the $\mathrm{CBH}$ and Kontsevich star products on $\mathrm{Sg}$ (see [3], [4]), i.e.

$$
D\left(f \star_{C B H} g\right)=(D f) \star_{K}(D g)
$$

for all $f, g \in S \mathfrak{g}$.

We also have

$$
\rho=e v_{0} \circ \exp \left(\sum_{n \geq 1} c_{2 n} \operatorname{tr}\left(\operatorname{ad}_{\partial}^{2 n}\right)\right)
$$

for yet undetermined constants $c_{2 n}$. Composing both sides of (17) with $\rho$ and using $(2)^{6}$ we obtain

$$
(\rho \circ D)\left(f \star_{C B H} g\right)=(\rho \circ D)(f)(\rho \circ D)(g) .
$$

\footnotetext{
${ }^{5}$ The map $D$ becomes the Duflo map when composed with $\exp \left(\operatorname{tr}\left(a d_{\partial}\right) / 4\right)$.

${ }^{6}$ The anomaly vanishes in this case.
} 


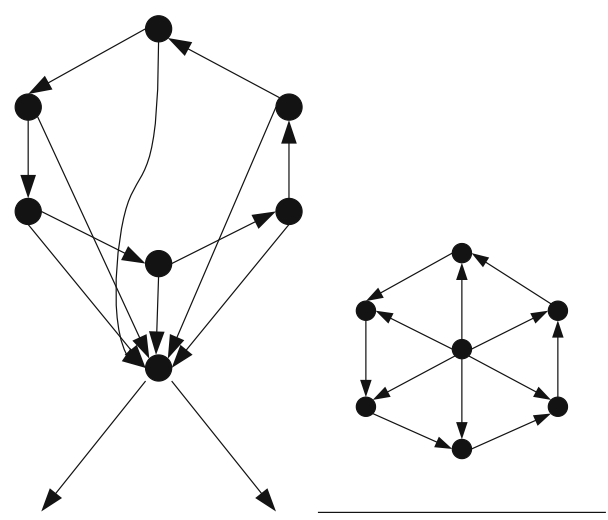

Figure 3. A typical anomaly wheel graph whose weight $c_{2 n}$ is computed in Theorem 18 (here $n=3$ ). To get a Cattaneo Felder wheel graph as in the proof of Theorem 18 one simply removes the two lower edges. The weight is the same as the Kontsevich weight of the graph on the right.

We want to show recursively that

$$
d_{2 n}:=\frac{B_{2 n}}{4 n(2 n) !}+c_{2 n}=0
$$

if $d_{2 j}=0$ for $j<n$. To do this pick $X \in \mathfrak{g}$ such that $\operatorname{tr}\left(\operatorname{ad}_{X}^{2 n}\right) \neq 0$ and set $f=g=$ $X^{n} .{ }^{7}$ Then a straightforward calculation proves the claim.

THEOREM 18. The $c_{2 n}$ computed in the preceding proof coincide with the weights of the anomaly wheel graphs as depicted in Figure 3, up to possibly signs. In particular, the weight of the anomaly graph of Figure 2 is nonzero.

Proof. Pick a Cattaneo Felder wheel graph $\Gamma$. See Figure 3 for an example. Let $\tilde{C}_{\Gamma}$ be the Cattaneo Felder configuration space as in Section 1.1. To divide out the scale invariance we will fix the central vertex of the wheel to lie on the unit quarter circle $\left\{e^{i t} ; t \in[0, \pi / 2]\right\}$ as depicted in Figure 4.

Consider the closed form $\tilde{\omega}_{\Gamma}$ defined on $\tilde{C}_{\Gamma}$ as in Section 1.1, and compute

$$
0=\int_{\tilde{C}_{\Gamma}} \mathrm{d} \tilde{\omega}_{\Gamma}=\int_{\partial \tilde{C}_{\Gamma}} \tilde{\omega}_{\Gamma}
$$

with the help of Stokes' theorem. There are several boundary strata contributing to the right-hand side. They correspond to center- or non-center-vertices

${ }^{7}$ One can always find a Lie algebra in which such an $X$ exists. The constants $c_{2 n}$ are weights of wheels and do not depend on the Lie algebra. 


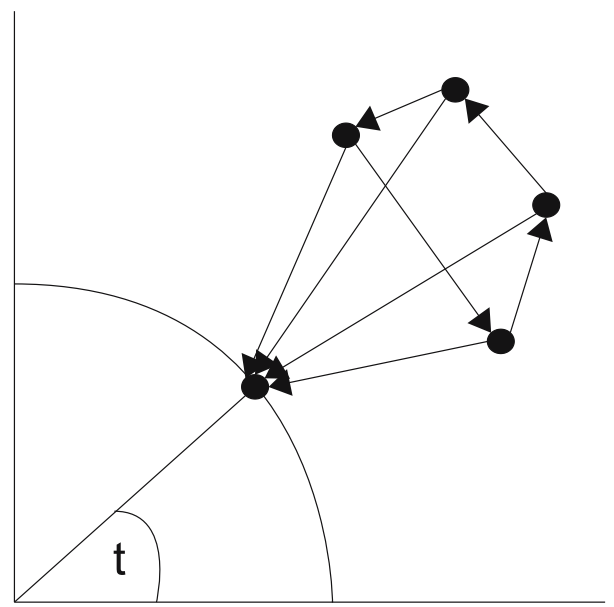

Figure 4. A typical configuration in $\tilde{C}_{\Gamma}$, where $\Gamma$ is the four-wheel graph. Note that the central vertex is confined to the quarter circle to divide out rescalings.

approaching the real axis, imaginary axis, or each other. We divide the strata into the following eight types, each treated separately: ${ }^{8}$

(i) If all vertices together approach the real axis and each other, the result is 0 by a result of Shoikhet [4].

(ii) If the central vertex approaches the real axis alone, the integral reduces to the integral of $\tilde{\omega}_{\Gamma}$ over $\tilde{C}_{\Gamma}$, yielding the Cattaneo Felder weight $\tilde{c}_{\Gamma}$.

(iii) If any subset of vertices approach the real axis and each other, except the two cases before, the result is zero by property 1 in Section 1.2. Note that there is always an edge from the collapsing "cluster" to the remainder of the graph.

(iv) If all vertices together approach the imaginary axis and each other the result is the Cattaneo Felder anomaly weight $\tilde{a}_{\Gamma}$ by property 3 and the algorithm for computing $\tilde{a}_{\Gamma}$.

(v) If any proper subset of vertices approach the imaginary axis, the result is zero by property 1 .

(vi) If more than two vertices come close to each other inside the quadrant, the result is zero by a lemma of Kontsevich.

(vii) If two non-center vertices come close to each other, the result is zero. This is because both are linked to the center vertex and hence the boundary integrand will contain a wedge product of at least twice the same one-form, i.e., zero.

\footnotetext{
${ }^{8}$ The readers not familiar with this kind of argument are referred to Kontsevich's proof of his theorem in [3].
} 
(viii) If any non-center vertex approaches the center vertex, the result is zero by similar reasoning as before. Note that automatically another vertex will be connected twice to the "cluster" of the two approaching vertices.

From this and the vanishing of the integral (18) the claim directly follows.

\section{References}

1. Cattaneo, A.S., Felder, G.: Relative formality theorem and quantisation of coisotropic submanifolds, (2005), arXiv:math.QA/0501540

2. Cattaneo, A.S., Felder, G.: Coisotropic submanifolds in Poisson geometry and branes in the Poisson sigma model. Lett. Math. Phys. 69, 157-175 (2004), arXiv:math.QA/ 0309180

3. Kontsevich, M.: Deformation quantization of Poisson manifolds, I. Lett. Math. Phys. 66, 157-216 (2003), arXiv:q-alg/9709040

4. Shoikhet, B.: Vanishing of the Kontsevich integrals of the wheels (2000), arXiv:math. QA/0007080

5. Weinstein, A.: The local structure of Poisson manifolds. J. Differ. Geom. 18, 523-557 (1983)

6. Willwacher, T.: Modules in deformation quantization, Diploma Thesis at ETH Zurich (2007) 